\title{
Epidemiology and antibiotic resistance trends of Pantoea species in a tertiary-care teaching hospital: A 12-year retrospective study
}

\author{
M GAJDÁCS ${ }^{1,2 *}$ \\ ${ }^{1}$ Department of Pharmacodynamics and Biopharmacy, Faculty of Pharmacy, University of Szeged, Szeged, Hungary \\ ${ }^{2}$ Institute of Clinical Microbiology, Faculty of Medicine, University of Szeged, Szeged, Hungary
}

(Received: September 5, 2019; revised manuscript received: October 26, 2019; accepted: November 6, 2019)

\begin{abstract}
Purpose: Pantoea species are pigmented, Gram-negative rods belonging to the Enterobacterales order. They are considered rare, opportunistic pathogens and are mostly implicated in nosocomial outbreaks affecting neonates and immunocompromised patients. The aim of this study was to describe the prevalence and antibiotic susceptibility of Pantoea species during a 12-year period. Materials and methods: This retrospective study was carried out using microbiological data collected between January 1, 2006 and December 31, 2017. Patients' data such as age, sex, inpatient/outpatient status, and empiric antibiotic therapy were also collected. Antimicrobial susceptibility testing was performed using E-tests; the interpretation was based on European Committee on Antimicrobial Susceptibility Testing breakpoints for Enterobacterales. Results: Seventy individual Pantoea spp. isolates were identified; the most frequently isolated species was Pantoea agglomerans. Most isolates were susceptible to relevant antibiotics. In 61 out of 68 patients, ampicillin was the empirically administered antibiotic. The highest levels of resistance were to amoxicillin-clavulanic acid and ampicillin. No extended spectrum beta-lactamase-positive isolate was detected. Conclusions: There is a scarcity of data available on the susceptibility patterns of Pantoea species, but our results correspond to what we could find in the literature. The development of multidrug-resistant (MDR) Gram-negative bacteria is a grave concern, and the development of MDR Pantoea spp. may be expected in the future.
\end{abstract}

Keywords: Pantoea, antibiotics, retrospective, epidemiology, resistance

\section{INTRODUCTION}

Pantoea species are pigmented, facultative anaerobic Gramnegative rods belonging to the Enterobacterales order and frequently isolated from natural environments (e.g., plants, soil, and water) [1, 2]. Pantoea agglomerans (previously known as Erwinia herbicola [1964] and Enterobacter agglomerans [1972]) is the most frequently isolated member of the genus in the context of human infections [3]. It is generally considered a rare, opportunistic pathogen with low virulence, mainly implicated in nosocomial outbreaks affecting neonates and immunocompromised patients $[4,5]$. Most of the affected patients in the literature had severe underlying illnesses (hematological malignancies or solid tumours, manifest AIDS); however, infections associated with penetrative trauma, contaminated intravascular catheters and fluids (dialysis and intravenous anesthesia), and agricultural occupation have also been reported $[1,4,6,7]$. In addition, associations of $P$. agglomerans bacteremia with antacid/proton pump inhibitor use (corresponding to a decreased protective effect of gastric acid) and gastrointestinal mucosal injury have been described, which may be attributable to the abundance of $P$. agglomerans on fruits or vegetables $[4,8]$.

The epidemiology and resistance trends of bacterial pathogens vary greatly by the profile of the healthcare institution and geographical region; therefore, the assessment of local data is essential to evaluate trends over time and to reflect on international data $[9,10]$. The spectrum of verified (i.e., clinically significant) bacterial pathogens has significantly broadened in recent years, due to the introduction of matrix-assisted laser desorption/ionization time-of-flight mass spectrometry (MALDI-TOF MS) and $16 \mathrm{~S}$ rRNA sequencing in the routine diagnostic workflow of microbiology laboratories [11]. The aim of this study was to describe the prevalence and antibiotic susceptibility patterns of Pantoea species isolated at a tertiary-care teaching hospital in Hungary during a 12-year period (2006-2017).

\section{MATERIALS AND METHODS}

\section{Study design and data collection}

The present retrospective study was based on microbiological data we collected between January 1, 2006 and

* Corresponding address: Márió Gajdács, PharmD, PhD; Department of Pharmacodynamics and Biopharmacy, Faculty of Pharmacy, University of Szeged, Eötvös utca 6, Szeged H-6720, Hungary; E-mail: mariopharma92@gmail.com

This is an open-access article distributed under the terms of the Creative Commons Attribution 4.0 International License, which permits unrestricted use, distribution, and reproduction in any medium, provided the original author and source are credited, a link to the CC License is provided, and changes - if any - are indicated. (SID_1) 
December 31, 2017 at the Institute of Clinical Microbiology (University of Szeged), the principal clinical microbiology laboratory of the Albert Szent-Györgyi Clinical Center in Szeged, Hungary. The Clinical Center has a bed capacity of 1,820 beds and annually serves more than 400,000 patients in the region, according to the data of the Hungarian National Health Insurance Fund (NEAK) [12]. Data were collected for samples positive $\left(10^{5}\right.$ or more CFU) for Pantoea species using the Institute's Laboratory Information System. Only the first isolate per patient was included in the study; however, isolates with different antibioticsusceptibility patterns were considered as different individual isolates [11]. Data on affected patients were also collected, limited to demographic characteristics (age, sex, and inpatient/outpatient status), indication for sample submission, and the administered empiric antibiotic therapy [13].

\section{Microbiological identification}

Sample processing in our Institute was carried out according to guidelines for routine clinical bacteriology, which have been previously described [13]. Between 2006 and 2012, the BD Bactec (Beckton Dickinson, Franklin Lakes, NJ, USA) detection system was employed in our Institute for the incubation of blood-culture bottles, while from 2013 onward, the BacT/ALERT 3D (bioMérieux, Marcyl'Étoile, France) detection system was used [14]. Bloodculture bottles were incubated for 5 days (21 days, if endocarditis was suspected) in the aforementioned detection systems. All culture media were incubated at $37{ }^{\circ} \mathrm{C}$ for $24-48 \mathrm{hr}$ in a $5 \% \mathrm{CO}_{2}$ atmosphere, 5\% sheep-blood agar, chocolate agar, and eosin methylene blue agar. Between 2006 and 2012, presumptive phenotypic (biochemical reaction-based) methods and VITEK 2 ID (bioMérieux, Marcy-l'Étoile) were used for bacterial identification, while after 2013, this was complemented by MALDI-TOF MS (Bruker Daltonik Gmbh, Germany). The methodology of sample preparation for MALDI-TOF MS measurements has been described elsewhere [14].

\section{Antibiotic susceptibility testing (AST)}

Antimicrobial susceptibility testing (AST) was performed for ampicillin, amoxicillin-clavulanic acid, chloramphenicol, ciprofloxacin, ceftriaxone, doxycycline, gentamicin, amikacin, meropenem, piperacillin-tazobactam, and sulfamethoxazole-trimethoprim, using E-test (Liofilchem, Abruzzo, Italy) on Mueller-Hinton agar plates. Interpretation of the results was based on European Committee on Antimicrobial Susceptibility Testing (EUCAST) breakpoints (http://www.eucast.org) for Enterobacterales. Staphylococcus aureus ATCC 29213, Enterococcus faecalis ATCC 29212, Proteus mirabilis ATCC 35659, Escherichia coli ATCC 25922, and Pseudomonas aeruginosa ATCC 27853 were used as quality-control strains. During data analysis, intermediate results were grouped and resistance was reported. Since 2011, if extended-spectrum beta-lactamase (ESBL) production was suspected, detection was carried out based on EUCAST recommendations (http://www.eucast.org/resistance_mechanisms/) using the AmpC-ESBL Detection Set (MAST Diagnostica GmbH, Reinfeld, Germany) [13].

\section{Statistical analysis}

Data consolidation and descriptive statistical analysis (including means or medians with ranges and percentages to characterize data) were performed using Microsoft Excel 2013 (Microsoft Corp., Redmond, WA, USA).

\section{RESULTS}

\section{Demographic characteristics and sample types}

The median age of affected patients was 40 years (range: $0.7-86$ years) with a female-to-male ratio of $0.84(45.59 \%$ female). The detailed age distribution of affected patients was the following: $0-17$ years, $n=10 ; 18-39$ years, $n=$ 18 ; 40-64 years $n=20$; and over 65 years, $n=18$. Inpatients were affected around 1.5 times more frequently than outpatients ( $n=41$ vs. $n=27$, respectively). Most of the isolates $(n=14)$ originated from blood cultures, abscesses $(n=9)$, and surgical samples $(n=7)$, followed by lower respiratory tract samples (trachea, bronchoalveolar lavage, and sputum), wound samples, and vaginal swabs $(n=6)$, gastric juice aspirates $(n=4)$, catheterized urine and central venous catheters $(n=3)$, urethral swabs, conjunctiva samples, high cervical swabs, inner ear samples, ejaculates, and nasal swabs ( $n=2$ each). In most cases, the reported empiric therapy was ampicillin $(n=61)$, followed by piperacillin/tazobactam $(n=2)$, amoxicillin/clavulanic acid, cefotaxime, cefuroxime, ceftazidime, and gentamicin $(n=1$ each $)$.

\section{Species distribution and susceptibility of Pantoea isolates}

Seventy individual Pantoea spp. isolates were identified during the 12-year period. The most frequently isolated species was $P$. agglomerans $(n=63)$, whereas other Pantoea species ( $P$. calida: $n=3 ; P$. septica, P. cypripedii, $P$. gaviniae, and $P$. anantis: $n=1$ each) were in a minority, and the identification of these isolates was after the introduction of MALDI-TOF MS (2013). All the isolates $(n=70)$ were susceptible to amikacin and meropenem. In addition, the overwhelming majority was susceptible to chloramphenicol, doxycycline, and gentamicin $(n=68)$, sulfamethoxazole-trimethoprim $(n=66)$, ceftriaxone $(n=64)$, and piperacillin-tazobactam $(n=62)$. The highest levels of resistance were recorded to amoxicillin-clavulanic acid $(n=28$ were susceptible) and ampicillin ( $n=12$ were susceptible). No ESBL-positive isolate was detected.

\section{DISCUSSION}

There is a scarcity of data available in the literature on the susceptibility patterns of Pantoea species, so additional 
data are necessary to provide a comprehensive picture of resistance trends. This picture would help in choosing an antimicrobial therapy and, ultimately, in preventing mortality from infections with Pantoea species [1-3]. In the Southern Great Plain of Hungary, most of the isolates were susceptible to the relevant antibiotics for treatment of Gram-negative infections. Aminopenicillins and amoxicillin-clavulanic acid were exceptions, for which $n=28$ and $n=12$ isolates were susceptible, respectively; this is clinically relevant information, as ampicillin was the most frequently administered antibiotic for the affected patients. Our results correspond to the findings of Cheng et al. (ampicillin susceptibility: 56\%, cefazolin susceptibility: 61\%, other antibiotics: $100 \%$ ), Cruz et al. (ampicillin susceptibility: $47.2 \%$, cefazolin susceptibility: $62.3 \%$, second-generation cephalosporins: $92.5 \%$, other antibiotics: $100 \%$ ), and other case studies reporting on Pantoea spp. infections [2-6, 8]. In the study of Mardaneh and Dallal [15], $12.5 \%$ of isolates were susceptible to ampicillin, $62.5 \%$ were susceptible to meropenem and amikacin, $75 \%$ were susceptible to gentamicin and piperacillin-tazobactam, and $100 \%$ were susceptible to colistin and tigecycline. In contrast, Büyükcam et al. [7] reported three multidrug-resistant (MDR) P. agglomerans strains in children, all of which were resistant to third-generation cephalosporins, carbapenems, ciprofloxacin, and aminoglycosides; the infections proved to be fatal in the affected patients. The development of MDR Gram-negative bacteria (particularly in the Enterobacterales order) is a grave concern in current clinical practice, and the development of Pantoea spp. resistant to several antibiotics may be expected in the future.

\section{CONCLUSIONS}

The aim of this study was to report the epidemiological characteristics and susceptibility patterns of Pantoea species in Hungary, over a long period of time (12 years). At the time of submission, there was no report in the literature describing the prevalence of Pantoea in Hungary. Clinical microbiology laboratories have pivotal roles in performing species-level identifications and in aiding physicians in the appropriate choice of empiric antibiotic therapy. A few drawbacks of this study need to be addressed. Medical records of the patients were not accessed; thus, the medical history and symptomatology of these patients were unknown. During the 12-year study period (between 2012 and 2013), the incubation system and the bacteriological identification methods were changed. In addition, several revisions of the EUCAST recommendations have been published. Finally, the resistance determinants of the strains described were not further assayed using polymerase chain reaction or sequencing technology. These factors may have distorted some of the results. For future reports, the aforementioned limitations could be addressed, especially the association of Pantoea isolation with clinical data/symptomatology (through the obtainment of patient data) and the use of molecular characterization or whole-genome sequencing (WGS) to characterize resistance determinants.
Acknowledgements: MG was supported by the National Youth Excellence Scholarship [grant number NTPNTFÖ-18-C-0225] and the ESCMID Mentorship and Observership Programme.

Author's contribution: MG conceived and designed the study, performed data collection and analysis, wrote, and revised the full paper.

Ethical approval: The study was deemed exempt from ethics review by the Institutional Review Board, and informed consent was not required as data anonymity was maintained.

Conflicts of Interest/Funding: The author declares no conflict of interest monetary, or otherwise.

\section{REFERENCES}

1. Dutkiewicz J, Mackiewicz B, Lemieszek KM, Golec M, Milanowski J. Pantoea agglomerans: a mysterious bacterium of evil and good. Part III. Deleterious effects: infections of humans, animals and plants. Ann Agric Environ Med. 2016;23(2):197-205.

2. Cruz AT, Cazacu AC, Allen CH. Pantoea agglomerans, a plant pathogen causing human disease. J Clin Microbiol. 2007;45(6):1989-92.

3. Siwakoti S, Sah R, Rajbhandari RS, Khanal B. Pantoea agglomerans infections in children: report of two cases. Case Rep Pediatr. 2018;2018:4158734.

4. Cheng A, Liu CY, Tsai HY, et al. Bacteremia caused by Pantoea agglomerans at a medical center in Taiwan, 2000-2010. J Microbiol Immunol Infect. 2013;46(3):187-94.

5. Mehar V, Yadav D, Sanghvi J, Gupta N, Singh K. Pantoea dispersa: an unusual cause of neonatal sepsis. Braz J Infect Dis. 2013;17(6):726-8.

6. Hagiya H, Otsuka F. Pantoea dispersa bacteremia caused by central line-associated bloodstream infection. Braz J Infect Dis. 2014;18(6):696-7.

7. Büyükcam A, Tuncer Ö, Gür D, et al. Clinical and microbiological characteristics of Pantoea agglomerans infection in children. J Infect Public Health. 2018;11(3):304-9.

8. Yamada K, Kashiwa M, Arai K, Satoyoshi K, Nishiyama H. Pantoea calida bacteremia in an adult with end-stage stomach cancer under inpatient care. J Infect Chemother. 2017;23(6):407-9.

9. Gajdács M, Urbán E. Epidemiological trends and resistance associated with Stenotrophomonas maltophilia bacteremia: a 10-year retrospective cohort study in a tertiary-care hospital in Hungary. Diseases. 2019;7(2):pii:E41.

10. Gajdács M, Urbán E. Prevalence and antibiotic resistance of Stenotrophomonas maltophilia in respiratory tract samples: a 10-year epidemiological snapshot. Health Serv Res Manager Epidemiol. 2019;15:6.

11. Gajdács M. Resistance trends and epidemiology of Aeromonas and Plesiomonas infections (RETEPAPI): a 10-year retrospective survey. Infect Dis. 2019;51(9):710-3. 
12. Nemzetközi Egészségbiztosítási Alapkezelő [National Health Insurance Fund of Hungary]. Kórházi ágyszám és betegforgalmi kimutatás 2017 [Hospital bed count and patient turnover report 2017] [Internet] [cited 8 July 2019]. Available from: http://www.neak.gov.hu/felso_menu/szakmai_oldalak/ publikus_forgalmi_adatok/gyogyito_megelozo_forgalmi_adat/ fekvobeteg_szakellatas/korhazi_agyszam.html

13. Gajdács M, Ábrók M, Lázár A, Burián K. Comparative epidemiology and resistance trends of common urinary pathogens in a tertiary-care hospital: a 10-year surveillance study. Medicina 2019;55(7):356.

14. Gajdács M. The relevance of anaerobic bacteria in brain abscesses: a ten-year retrospective analysis (2008-2017). Infect Dis (London). 2019;51(10):779-81.

15. Mardaneh J, Dallal MM. Isolation, identification and antimicrobial susceptibility of Pantoea (Enterobacter) agglomerans isolated from consumed powdered infant formula milk (PIF) in NICU ward: first report from Iran. Iran J Microbiol. 2013;5(3):263-7. 